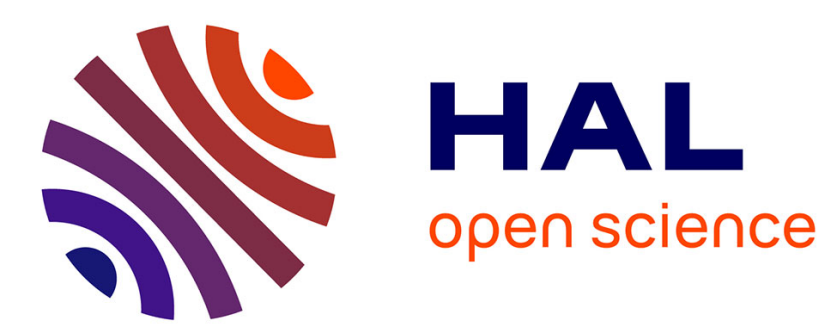

\title{
Decomposition of the different contributions to permittivity, losses, and tunability in BaSrTiO3 thin films using the hyperbolic law
}

Kevin Nadaud, Caroline Borderon, Raphaël Renoud, Hartmut W. Gundel

\section{- To cite this version:}

Kevin Nadaud, Caroline Borderon, Raphaël Renoud, Hartmut W. Gundel. Decomposition of the different contributions to permittivity, losses, and tunability in BaSrTiO3 thin films using the hyperbolic law. Journal of Applied Physics, 2016, 119 (11), pp.114101. 10.1063/1.4943935 . hal-01307944

\section{HAL Id: hal-01307944 \\ https://hal.science/hal-01307944}

Submitted on 27 Apr 2016

HAL is a multi-disciplinary open access archive for the deposit and dissemination of scientific research documents, whether they are published or not. The documents may come from teaching and research institutions in France or abroad, or from public or private research centers.
L'archive ouverte pluridisciplinaire HAL, est destinée au dépôt et à la diffusion de documents scientifiques de niveau recherche, publiés ou non, émanant des établissements d'enseignement et de recherche français ou étrangers, des laboratoires publics ou privés. 


\title{
Decomposition of the different contributions to permittivity, losses, and tunability in $\mathrm{BaSrTiO}_{3}$ thin films using the hyperbolic law
}

\author{
Kevin Nadaud, Caroline Borderon, ${ }^{\text {a) }}$ Raphaël Renoud, and Hartmut W. Gundel \\ IETR, UMR CNRS 6164, University of Nantes, Nantes F-44322, France
}

(Received 18 November 2015; accepted 1 March 2016; published online 15 March 2016)

\begin{abstract}
In this paper, the different contributions to the permittivity of a $1 \%$ manganese-doped $\mathrm{BaSrTiO}_{3}$ thin film are presented as a function of the applied DC field. The hyperbolic law has been used to discern the lattice, domain wall vibration, and pinning/unpinning contributions. This decomposition permits us to study the weight of the respective contribution in the total permittivity, the losses, and the tunability. By determining the figure of merit $(F o M)$ of each contribution, the ratio between tunability and losses, it is possible to identify the phenomenon which should be limited or enhanced in order to optimize the material's dielectric properties. It is shown that the tunability of the domain wall contribution (approximately 80\%) is very important compared to the lattice contribution (41\%), the associated dissipation factor, however, is also much larger (0.2 instead of 0.014). Even if the domain wall contribution has been shown to be weak in the investigated thin film (less than $3 \%$ in permittivity and tunability), the weight of the losses is not negligible (around 18\%). Hence, the domain contribution has to be limited in order to conserve a high FoM for the material. Moreover, it is shown that the AC field used for the material's characterization is important because it governs the weight of the domain wall losses and thus the FoM. (C) 2016 AIP Publishing LLC.

[http://dx.doi.org/10.1063/1.4943935]
\end{abstract}

\section{INTRODUCTION}

Since many years, ferroelectric thin films are investigated for their high-k character and the tunable permittivity under a DC bias field $E_{D C}$. Amongst others, this allows the realization of tunable microwave devices. ${ }^{1-3}$ However, such applications rely on a large variation of the permittivity as a function of the bias field and on low dielectric losses $\left(\tan \delta<10^{-2}\right)$. Many studies have been conducted in order to improve the dielectric properties of these materials and more particular its dielectric losses. The material is often placed in a MIM (Metal-Insulator-Metal) geometry. The real part of the permittivity thus can be deduced from the capacity value, whereas the imaginary part is obtained from the dielectric losses $\tan \delta=\varepsilon_{r}^{\prime \prime} / \varepsilon_{r}^{\prime}$ which represent the delay of the dielectric displacement with respect to the electric field. The applied field is an alternating field of frequency $f$ and amplitude $E_{A C}$. While the measuring frequency is regularly reported, only few studies mention the amplitude of the used field. However, this indication is important, since ferroelectrics have a non-linear response due to the presence of domain wall motions, even at low AC fields. Thus, the complex permittivity (real and imaginary parts) depends on the field amplitude $E_{A C}$ and follows a hyperbolic law: ${ }^{4,5}$

$$
\varepsilon_{r}=\varepsilon_{r l}+\sqrt{\varepsilon_{r-r e v}^{2}+\left(\alpha_{r} E_{A C}\right)^{2}},
$$

where $\varepsilon_{r l}$ corresponds to the lattice contribution, $\varepsilon_{r-r e v}$ to the contribution due to domain wall vibrations (also called reversible contribution), and $\alpha_{r}$ to the contribution due to domain wall pinning/unpinning (also called irreversible

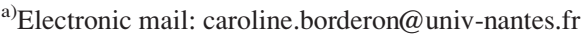

contribution). $\varepsilon_{r-r e v}$ and $\alpha_{r}$ depend on the crystal structure, but $\alpha_{r}$ also reflects the presence of impurities, dopants, or defects. ${ }^{6,7}$ In addition, the different parameters are frequency dependent. ${ }^{5,7}$ According to the $\alpha_{r}$ value, the permittivity can be more or less sensitive to the measuring field $E_{A C}$. Therefore, it is mandatory to know the weight of each contribution to the permittivity. Moreover, the domain wall parameters $\varepsilon_{r-r e v}$ and $\alpha_{r}$ depend on the material's polarization state and hence may contribute differently when the electric field $E_{D C}$ varies. Those parameters are, by definition, independent from the amplitude of the AC field. This description is only valid for AC field lower than $E_{c} / 2,{ }^{4}$ and for higher field a more complete description, including switching effect, is necessary. At AC fields higher than the coercive field, saturation phenomenon may appear, and those parameters should depend on the amplitude of the AC field. Moreover, this case is not aborded here.

Thus, in the present paper, the different contributions to the permittivity of a $\mathrm{Ba}_{0.80} \mathrm{Sr}_{0.20} \mathrm{TiO}_{3}$ (BST) thin film are presented as a function of the DC bias field. In order to separate the different contributions, the real and imaginary parts of the overall permittivity have been measured as a function of the AC electric field. Then, application of the hyperbolic law allows depiction of the evolution of each contribution and its respective permittivity as a function of the material's polarization state. Generalizing the usual definition, ${ }^{8}$ the tunability of each contribution can be calculated

$$
n_{r-\varepsilon}=\frac{\varepsilon\left(E_{c}\right)-\varepsilon(E)}{\varepsilon\left(E_{c}\right)} \times 100,
$$

where $\varepsilon\left(E_{c}\right)$ and $\varepsilon(E)$ are the considered contributions to the permittivity $\left(\varepsilon_{r l}, \varepsilon_{r-r e v}\right.$, or $\left.\alpha_{r}\right)$, respectively, at the coercive 
field and under the applied bias electric field $E$. To evaluate the dissipative behavior of each contribution, we define a generalized dissipation factor

$$
m_{\varepsilon}=\frac{\varepsilon^{\prime \prime}}{\varepsilon^{\prime}},
$$

with $\varepsilon^{\prime \prime}$ and $\varepsilon^{\prime}$, respectively, the imaginary and the real parts of the considered phenomena. Note that in the case of the material's overall permittivity, the dissipation factor corresponds to the loss factor $\tan \delta$. This permits us to define the figure of merit $(F O M)$ of each contribution, using the classical definition ${ }^{8}$

$$
F_{O} M_{\varepsilon}=\frac{n_{r-\varepsilon}}{m_{\varepsilon}\left(E_{c}\right) \times 100},
$$

where $m_{\varepsilon}\left(E_{c}\right)$ corresponds to the dissipation factor, at the coercive field, given in absolute values to correspond to the delay between the applied field and the electric displacement. For the calculation of the $F o M, m_{\varepsilon}\left(E_{c}\right)$ is multiplied by 100 in order to obtain homogeneity with the tunability expression (given in percent). The FoM is used for quantifying the compromise between high tunability and low losses. The higher the figure of merit, the more beneficial is the respective contribution to the overall material's properties.

\section{EXPERIMENTS}

The $\mathrm{Ba}_{0.80} \mathrm{Sr}_{0.20} \mathrm{TiO}_{3}$ thin films have been realized by a sol-gel process based on the use of an alkoxide precursor., ${ }^{7,9}$ The solution has been deposited on platinum coated alumina substrates at $4000 \mathrm{rpm}$ during $20 \mathrm{~s}$, and the samples have been annealed during $15 \mathrm{~min}$ in a pre-heated open air furnace at $750{ }^{\circ} \mathrm{C}$. Deposition of fourteen layers results in an overall film thickness of typically $0.85 \mu \mathrm{m}$. The BST thin films have been doped with $1 \%$ of manganese corresponding to a $\mathrm{Ba}_{0.80} \mathrm{Sr}_{0.20} \mathrm{Ti}_{0.99} \mathrm{Mn}_{0.01} \mathrm{O}_{3}$ composition. This $1 \%$ dopant rate has been found to be optimum ${ }^{7}$ for reducing the leakage current, the dielectric losses, and the low frequency charge carrier diffusion. ${ }^{7,10,11}$ Platinum top electrodes have been deposited by RF sputtering in order to realize a MIM capacitor. The capacitance and the dielectric loss factor $(\tan \delta)$ have been measured using an Agilent 4294A impedance meter at $100 \mathrm{kHz}$. To change the polarization state of the material, a DC electric field has been superposed to the alternating field. For a fixed DC field, the AC field amplitude sweeps from $0.06 \mathrm{kV} / \mathrm{cm}$ to $12 \mathrm{kV} / \mathrm{cm}$ (corresponding, respectively, to $5 \mathrm{mV}$ and $1 \mathrm{~V}$ ). The procedure is repeated for each DC field, which permits us to obtain the full $\varepsilon_{r}-E$ loop. The value of the DC field varies from $-230 \mathrm{kV} / \mathrm{cm}$ to $230 \mathrm{kV} / \mathrm{cm}$ (corresponding to $\pm 20 \mathrm{~V}$ ) with an increment of $11.8 \mathrm{kV} / \mathrm{cm}(1 \mathrm{~V}$ on a thickness of $0.85 \mu \mathrm{m})$ on every $120 \mathrm{~s}$. All the measurements were done at room temperature $\left(20^{\circ} \mathrm{C}\right)$.

\section{RESULTS AND DISCUSSION}

The X-ray diffraction pattern of the studied BST thin film is shown in Fig. 1. The (100), (110), (111), (200), and (211) peaks, corresponding to the perovskite structure of the

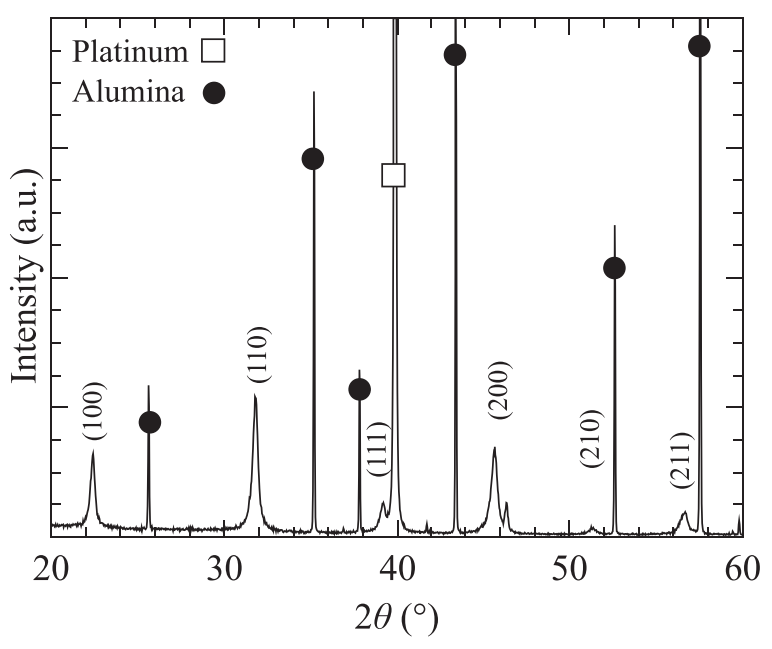

FIG. 1. X-ray diffraction pattern of the BST thin film.

material, indicate a non-textured polycrystalline structure without preferential orientation. No parasitic peaks and no evidence for any secondary phase formation are visible.

The polarization hysteresis cycle of the thin film is shown in Fig. 2. The very slim shaped loop indicates an only very small hysteresis effect, which is typical for soft materials like BST thin films. ${ }^{12}$

\section{A. Influence of the AC field on the material's overall permittivity}

The relative permittivity and the dielectric losses, measured at $100 \mathrm{kHz}$, are shown in Fig. 3 as a function of the DC bias field for two different $\mathrm{AC}$ measuring fields. Whereas the $\mathrm{AC}$ field almost does not impact the permittivity value, the influence on the dielectric losses of the material is clearly visible. This results from the presence of the irreversible domain wall motion contribution $\alpha_{r}$, also called domain wall pinning/unpinning which depends on the AC field. ${ }^{4,5}$ This phenomenon is more important for low DC bias fields, suggesting that the domain wall contribution depends on the polarization state. Consequently, the tunability and the $F o M$ are affected, too. Under a low AC field $\left(E_{A C}=0.06 \mathrm{kV} / \mathrm{cm}\right)$, the tunability is $40.8 \%$, and the losses $\tan \delta$ are 0.0146 ,

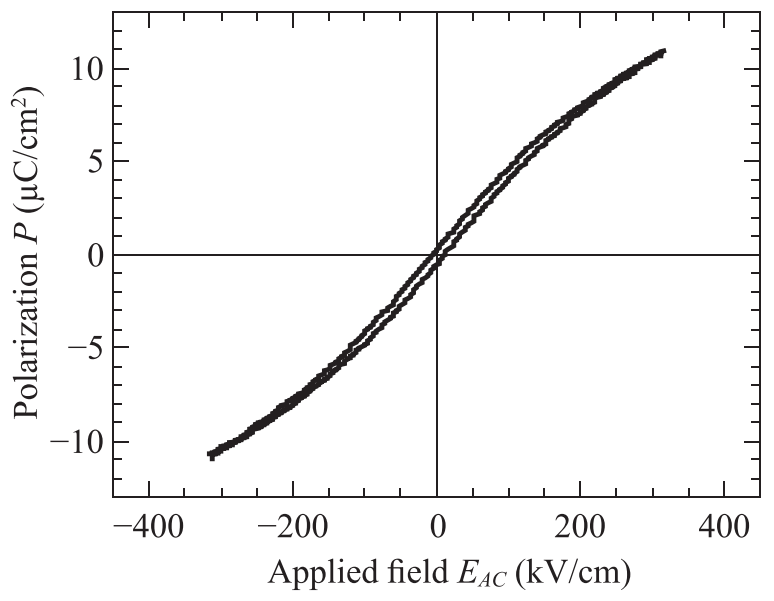

FIG. 2. Polarization as a function of the AC field, measured at $50 \mathrm{~Hz}$. 


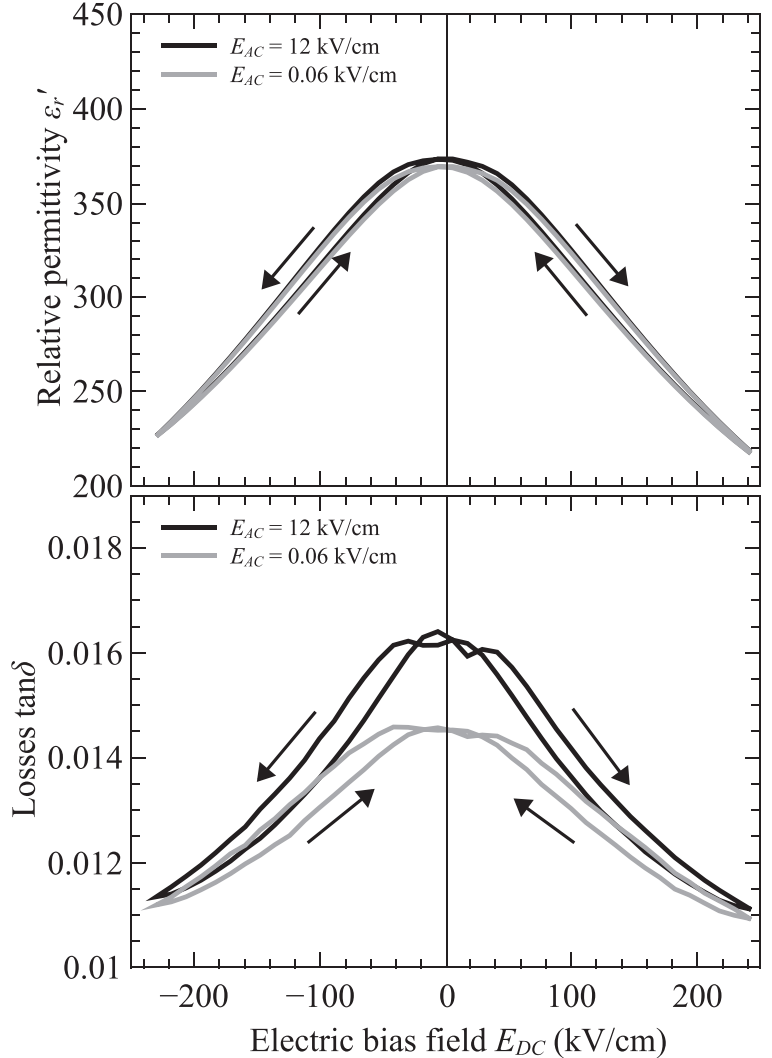

FIG. 3. Overall relative permittivity and dielectric losses as a function of the applied DC field, for two AC field values, measured at $100 \mathrm{kHz}$.

which corresponds to a FoM of 27.9, whereas at $E_{A C}=12 \mathrm{kV} / \mathrm{cm}$ the tunability and the losses are $41.3 \%$ and 0.0163 , respectively, and a lower FoM (25.4) is obtained. As a consequence, even if the influence of the domain wall motion on the permittivity and the tunability seems quite small, the effect on the dielectric losses and the FoM is not negligible. This shows the importance of the AC characterization field and the need to examine the domain wall contribution. Moreover, when using the thin film for an application, the magnitude of the operating AC field will impact the material's properties as well and hence the performance of the device. Thus, it is necessary to characterize the material under realistic application conditions.

To obtain the characteristic parameters, the evolution of the real and imaginary parts of the permittivity as a function of the applied bias field can be described by a Lorentzian distribution, which seems to be an appropriate trial function according to Wang ${ }^{13}$ or Jo. ${ }^{14}$ Thus, we define

$$
f(E)=\frac{A_{\max }-A_{\infty}}{1+\left(\frac{E \pm E_{c}}{w}\right)^{2}}+A_{\infty},
$$

where $E_{c}$ is the coercive field, $A_{\max }$ is an amplitude factor corresponding to the maximum value of the permittivity, and $w$ is the full width at half maximum of the Lorentzian. $A_{\infty}$ is the permittivity at an infinite applied bias field. Fitting of the relative permittivity is shown in Fig. 4. The Lorentzian function describes quite well the evolution of the relative permittivity and may be used for modeling the material in a circuit

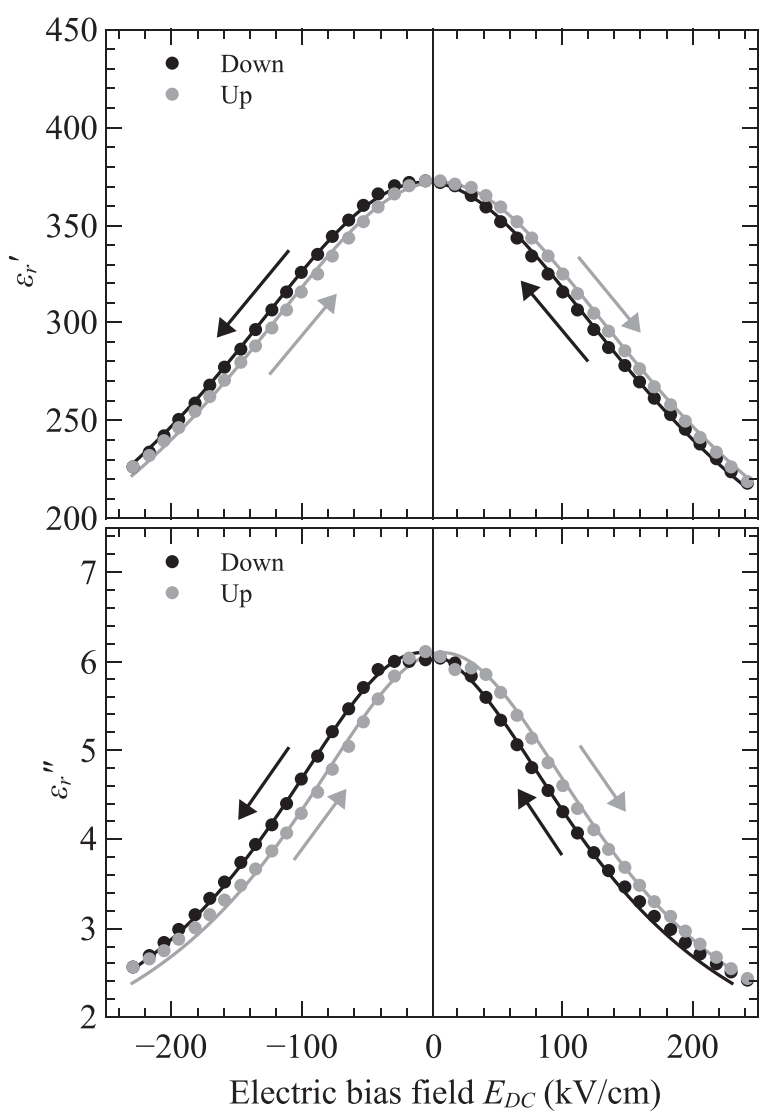

FIG. 4. Real and imaginary parts of the overall permittivity as a function of the applied DC field, measured at $100 \mathrm{kHz}$ for an AC field of $12 \mathrm{kV} / \mathrm{cm}$ and fit of the experimental data with the Lorentzian function (5).

simulator, for example. The obtained coefficients are $A_{\max }^{\prime}$ $=373, A_{\infty}^{\prime}=102, E_{c}^{\prime}=5 \mathrm{kV} / \mathrm{cm}$, and $w^{\prime}=210 \mathrm{kV} / \mathrm{cm}$ for the real part and $A_{\text {max }}^{\prime \prime}=6.1, A_{\infty}^{\prime \prime}=0.9, E_{c}^{\prime \prime}=8 \mathrm{kV} / \mathrm{cm}$, and $w^{\prime \prime}=150 \mathrm{kV} / \mathrm{cm}$ for the imaginary part, for an AC field of $12 \mathrm{kV} / \mathrm{cm}$. For the BST thin film studied, the characteristic parameters for the AC field of $0.06 \mathrm{kV} / \mathrm{cm}$ do not really differ. This is due to the fact that there is only a weak contribution of domain wall motions which shall be pointed out in more detail further below. Fig. 5 shows the Lorentzian fit of the overall dielectric losses as a function of the applied DC field which

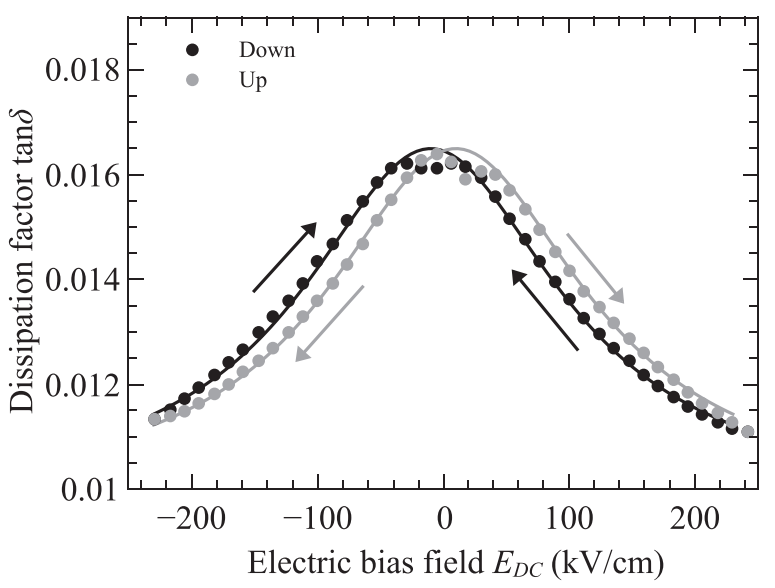

FIG. 5. Overall dielectric losses as a function of the applied DC field, measured at $100 \mathrm{kHz}$ for an $\mathrm{AC}$ field of $12 \mathrm{kV} / \mathrm{cm}$ and fit of the experimental data with the Lorentzian function (5). 
describes quite well the evolution. The coefficients are $A_{\text {max }-m}^{\prime}=0.014, A_{\infty-m}^{\prime}=0.010, E_{c-m}^{\prime}=15 \mathrm{kV} / \mathrm{cm}$, and $w_{m}^{\prime}$ $=160 \mathrm{kV} / \mathrm{cm}$.

The AC field dependence of the permittivity for two values of the DC field amplitude is shown in Fig. 6. At low DC bias fields $\left(E_{D C}=0 \mathrm{kV} / \mathrm{cm}\right)$ near to the coercive field, the permittivity increases with the AC measuring field due to the domain wall pinning/unpinning contribution and follows an hyperbolic law. At higher bias fields $\left(E_{D C}=230 \mathrm{kV} / \mathrm{cm}\right)$, the permittivity is only slightly affected, showing that the domain wall contribution is very small. At $E_{D C}=0 \mathrm{kV} / \mathrm{cm}$, the parameter $\alpha_{r}$ of the hyperbolic law is equal to $0.43 \mathrm{~cm} / \mathrm{kV}$, whereas it is only $0.06 \mathrm{~cm} / \mathrm{kV}$ at $E_{D C}=230 \mathrm{kV} / \mathrm{cm}$. This clearly reflects the fact that the DC bias field and thus the polarization state of the ferroelectric thin film affect the domain wall contribution to the permittivity. The effect of the DC field on the AC field dependence of the permittivity is also visible on the imaginary part of the permittivity (Fig. 6), where an increase is well visible when the DC field is low $\left(E_{D C}=0 \mathrm{kV} / \mathrm{cm}\right)$ and almost no increase is visible when the DC field is higher $\left(E_{D C}=230 \mathrm{kV} / \mathrm{cm}\right)$. While the effect of the domain wall motion on the material's permittivity is quite low (around 1\% of variation), it is not negligible on the imaginary part of the permittivity (and thus the losses), since it represents a variation of approximately $15 \%$. This is well visible from Fig. 3, where a large difference in permittivity and losses between the two AC values can be seen at lower DC fields, too.

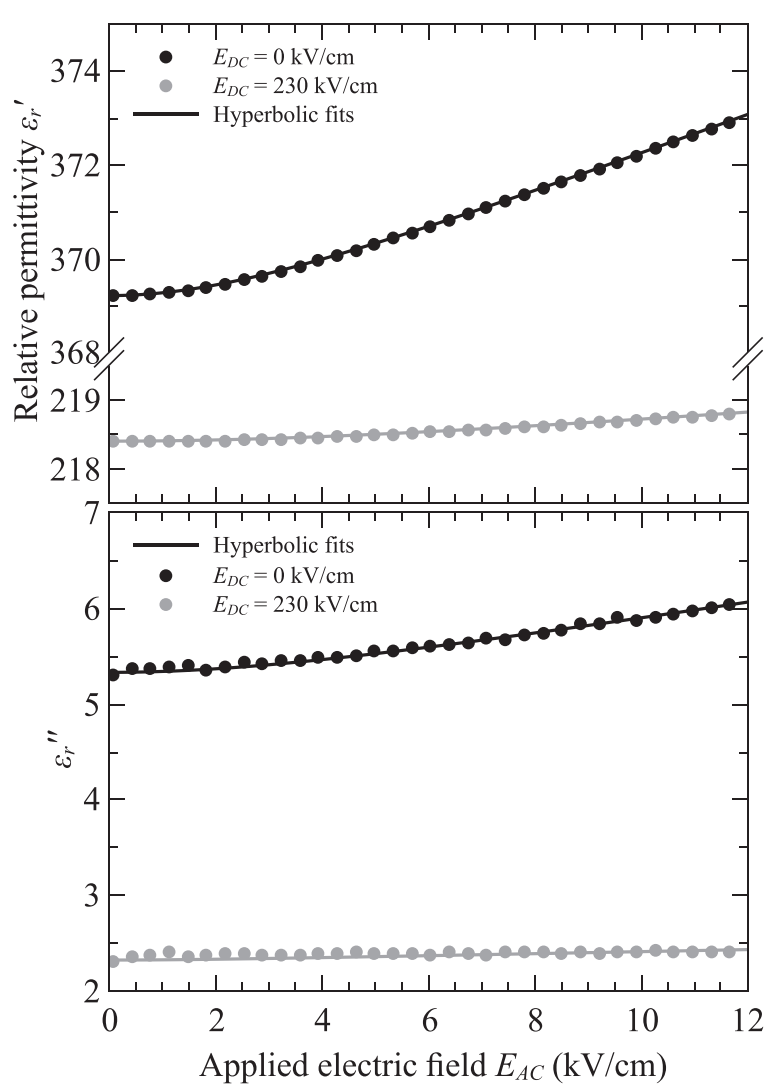

FIG. 6. Overall relative permittivity at $100 \mathrm{kHz}$ under $0 \mathrm{kV} / \mathrm{cm}$ and $230 \mathrm{kV} /$ $\mathrm{cm} \mathrm{DC}$ bias field as a function of the applied AC measuring field.

\section{B. Study of the different contributions to the permittivity}

To describe the evolution of the domain wall contributions as a function of the polarization state, the real and the imaginary parts of the permittivity have been measured as a function of amplitude of the AC field for different DC bias fields. The extraction according to the hyperbolic law has been done for a maximum bias field of $\pm 230 \mathrm{kV} / \mathrm{cm}$ for increasing and decreasing voltage in order to obtain the characteristic butterfly loop. First, the domain wall pinning/ unpinning will be studied, since the bias field affects the slope of the permittivity (Fig. 6), then, the domain wall vibration and the lattice parameters are presented. The relative weight of the different contributions shall be discussed in Section III C.

\section{Domain wall pinning/unpinning contribution}

The domain wall pinning/unpinning parameter as a function of the applied DC field is presented in Fig. 7. It can be seen that the real and imaginary parts describe a (rather closed) butterfly loop, decrease when the bias field increases due to the domains' coalescence, ${ }^{15}$ and tend to zero at highest applied fields. As the number of domains decreases when the applied DC field increases, the domain wall density also becomes smaller. The domain wall pinning/unpinning depends on the domain wall density, and hence this contribution is reduced at higher DC fields. Fig. 6 well illustrates this phenomenon as the parameter $\alpha_{r}$, corresponding to the slope

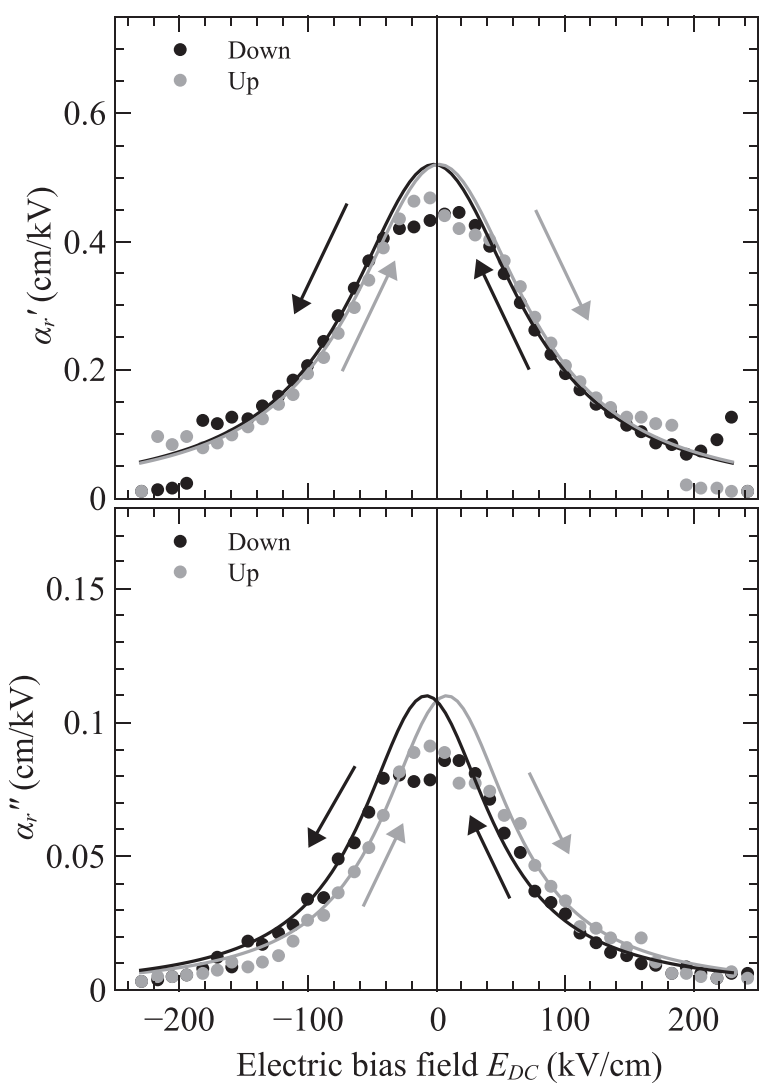

FIG. 7. Real and imaginary parts of the domain wall pinning/unpinning contribution to the permittivity as a function of the applied DC field at $100 \mathrm{kHz}$. 
TABLE I. Fitting coefficients for the different contributions (domain wall pinning/unpinning, domain wall vibration, and lattice contributions) to the overall dielectric permittivity. $E_{c}$ and $w$ are given in $\mathrm{kV} / \mathrm{cm}$, and $A_{\max -\alpha}$ and $A_{\infty-\alpha}$ which refer to the domain wall pinning/unpinning are given in $\mathrm{cm} / \mathrm{kV}$. For the overall permittivity fits, the values are given for an AC field of $12 \mathrm{kV} / \mathrm{cm}$.

\begin{tabular}{|c|c|c|c|c|c|c|c|c|c|c|c|c|}
\hline \multirow[b]{2}{*}{ Contribution } & \multicolumn{4}{|c|}{ Real part } & \multicolumn{4}{|c|}{ Imaginary part } & \multicolumn{4}{|c|}{ Dissipation factor } \\
\hline & $A_{\max }^{\prime}$ & $A_{\infty}^{\prime}$ & $E_{c}^{\prime}$ & $w^{\prime}$ & $A_{\max }^{\prime \prime}$ & $A_{\infty}^{\prime \prime}$ & $E_{c}^{\prime \prime}$ & $w^{\prime \prime}$ & $A_{\max -m}$ & $A_{\infty-m}$ & $E_{c-m}$ & $w_{m}$ \\
\hline$\alpha_{r}$ (pinning/unpinning) & 0.52 & 0 & 5 & 80 & 0.11 & 0 & 8 & 60 & 0.20 & 0.025 & 10 & 150 \\
\hline$\varepsilon_{r-\text { rev }}($ vibration $)$ & 1.70 & 0 & 5 & 130 & 0.49 & 0 & 8 & 70 & 0.330 & $\sim 0$ & 5 & 90 \\
\hline$\varepsilon_{r l}$ (lattice) & 367 & 102 & 5 & 210 & 5.05 & 0.9 & 8 & 185 & 0.014 & 0.010 & 15 & 160 \\
\hline Overall permittivity $(12 \mathrm{kV} / \mathrm{cm})$ & 373 & 102 & 5 & 210 & 6.1 & 0.9 & 8 & 150 & 0.0165 & 0.010 & 10 & 125 \\
\hline
\end{tabular}

of the hyperbolic law, depends on the DC bias field. The tunability of the domain wall pinning/unpinning contribution has been calculated using (2) and is $n_{r-\alpha}=88 \%$ under a bias field of $230 \mathrm{kV} / \mathrm{cm}$. This value is very high compared to the overall material's tunability $(41 \%)$.

As for the overall permittivity, the real and imaginary parts of $\alpha_{r}$ have been fitted according to the Lorentzian distribution, and the coefficients are given in Table I. Even if there is some noise, the fitting function describes quite well the evolution of the domain wall pinning/unpinning contribution as a function of the DC field. The value of $A_{\max -\alpha}^{\prime}$ is $0.52 \mathrm{~cm} /$ $\mathrm{kV}$ and is smaller than reported for other materials like Lead Zirconate Titanate (PZT). ${ }^{16,17}$ The domain wall density seems to be low which might be due to the small grain size. ${ }^{7}$ The value of $A_{\infty-\alpha}^{\prime}$ and $A_{\infty-\alpha}^{\prime}$ is null, which signifies that there is no domain wall pinning/unpinning contribution when the field is infinite. At infinite field, the domain wall density tends to zero, since all the domains are oriented in the same direction (polarization saturation). As a consequence, the tunability of the domain wall pinning/unpinning parameter is $100 \%$ for an infinite field. The coercive field of the real and imaginary parts is slightly different, indicating that the DC field does not affect them in the same way. This difference of sensitivity to the DC field can also be seen on the width of the Lorentzian fit $w_{\alpha}$ which is smaller for the imaginary part, indicating that the losses are more influenced by the DC field.

The dissipation factor of the domain wall jump $m_{\alpha}$ is reported in Fig. 8 as a function of the DC bias field. The

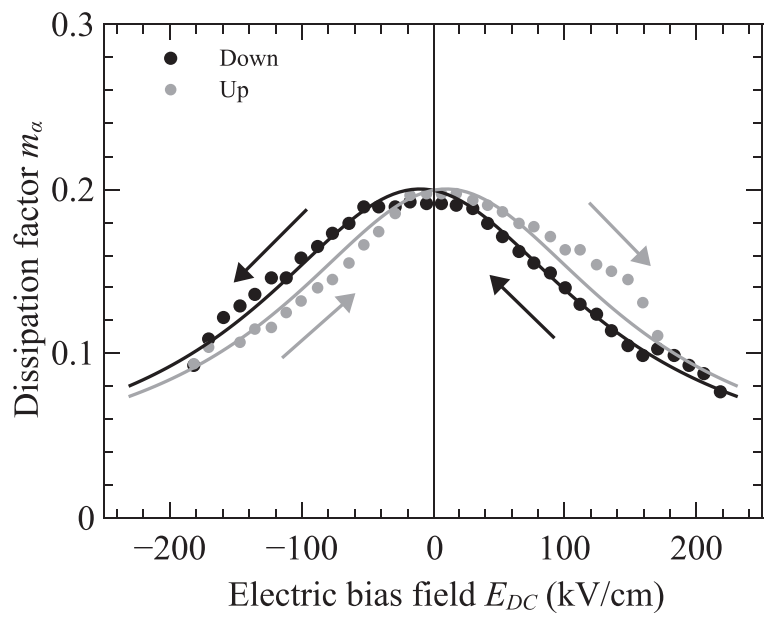

FIG. 8. Dissipation factor of the domain wall pinning/unpinning contribution to the permittivity as a function of the applied DC field at $100 \mathrm{kHz}$. maximum value of $m_{\alpha}$ is 0.2 which is smaller than reported elsewhere for PZT. ${ }^{18}$ This is due to a low domain wall density that induces only little interaction between the walls. ${ }^{7}$ The dissipation factor decreases as the DC bias field increases. The evolution is similar to this observed for the real and imaginary parts of $\alpha_{r}$; thus, the same fit has been used to describe the experimental data. The different coefficients derived for the domain wall pinning/unpinning phenomenon are given in Table I.

Contrary to the coefficient of the real and imaginary parts of $\alpha_{r}$, the $m_{\alpha}$ value for an infinite DC field is not zero. The finite limit value may correspond to the needed energy for a domain wall to jump from one defect to another. As the DC bias field decreases, the domain wall density increases, ${ }^{15}$ as well as the dissipation factor (Fig. 8). This increase may come from the interaction between the domain walls, which is more pronounced when the domain wall density is high. ${ }^{7}$ As a consequence, the domain wall pinning/unpinning dissipation factor has two contributions, the influence between the domain walls which depends on the domain wall density and the intrinsic losses caused by the energy needed in order to jump from one defect to another.

From the material's application point of view, it is also important to note that the maximum value of the dissipation factor is 0.2 , which indicates that the pinning/unpinning is a very dissipative phenomenon compared to the dielectric losses of the overall permittivity (17 times higher). Even if the tunability of the parameter $\alpha_{r}^{\prime}$ is very high $\left(n_{r-\alpha}=88 \%\right.$ in our case) and tends to $100 \%$ for an infinite field, this contribution has to be reduced in order to limit the overall losses of the material because its figure of merit is very low $\left(F o M_{\alpha}=4.18\right)$. This illustrates well that a high tunability may be related to very high losses, and it is hence essential to calculate the FoM which shows the compromise between high tunability and low losses.

\section{Domain wall vibration contribution}

The second contribution of the domain walls to the complex dielectric permittivity is its vibration which is represented by the parameter $\varepsilon_{r-r e v}$. The real and imaginary parts of $\varepsilon_{r-\text { rev }}$ as a function of the DC bias field are shown in Fig. 9. As this contribution is weak compared to the overall permittivity (around 1.5 vs 370) and the data are noisy, the extraction of the parameters is quite difficult. The real and imaginary parts of $\varepsilon_{r-r e v}$ show the same evolution than $\alpha_{r}$, which is again due to the domains' density which decreases 


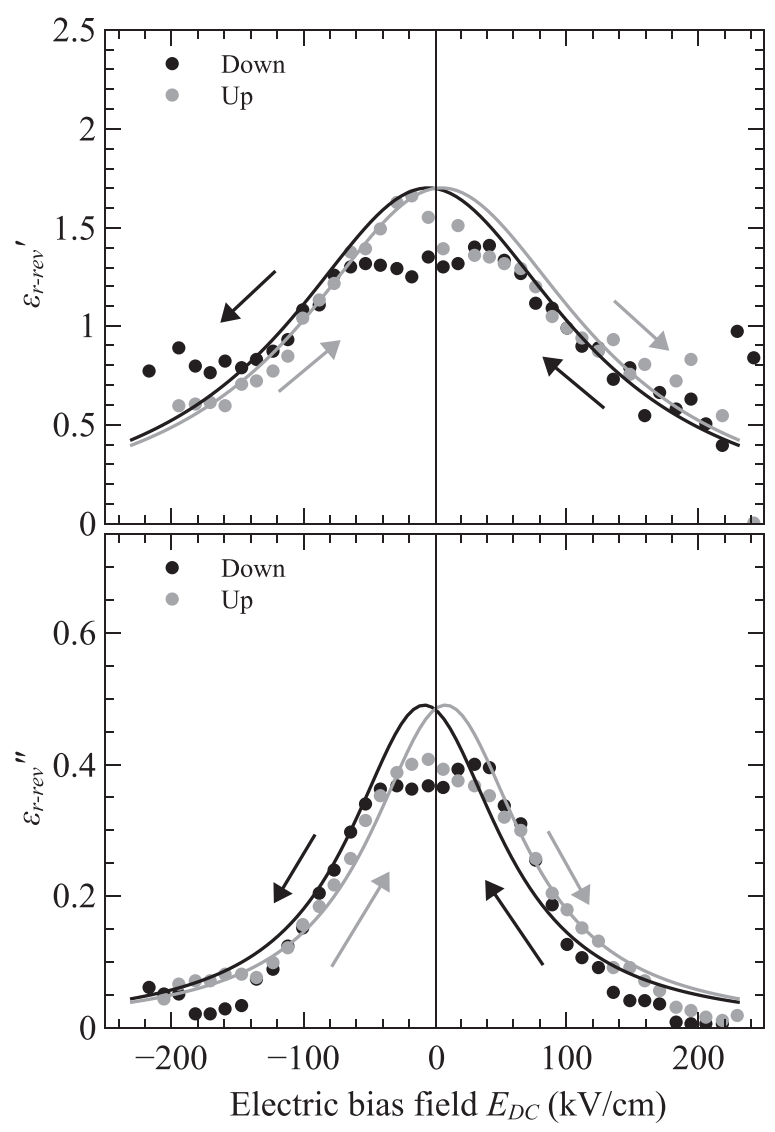

FIG. 9. Real and imaginary parts of the domain wall vibration contribution to the permittivity as a function of the applied DC field at $100 \mathrm{kHz}$.

when the DC field is increased. The tunability of the domain wall vibration contribution, calculated from Equation (2), is $77 \%$ at a bias field of $230 \mathrm{kV} / \mathrm{cm}$, which is again very high. This is due to the fact that the number of domain walls is strongly influenced by the DC field. The Lorentzian distribution also has been used in order to fit the evolution of the domain wall vibration contributions, and the coefficients are given in Table I. Even though there is some noise, it can be seen from Fig. 9 that the proposed fit describes well the real and imaginary parts of $\varepsilon_{r-r e v}$. Likewise the pinning/unpinning contribution, the imaginary part decreases faster than the real part when a DC field is applied, which corresponds to a smaller width $w_{r e v}^{\prime \prime}$. The coercive field $E_{c-\text { rev }}$ also has a similar value than this for $\alpha_{r}$. At infinite field, $\varepsilon_{r-r e v^{\prime}}$ and $\varepsilon_{r-r e v^{\prime \prime}}$ are again zero due to the domain wall density which tends to zero. Similarly to the pinning/unpinning contribution, the tunability of the domain wall vibration contribution is $100 \%$ for an infinite DC bias field.

To quantify the losses due to the domain wall vibration phenomenon, the dissipation factor $m_{\text {rev }}$ has been calculated and is presented as a function of the applied bias field in Fig. 10. It decreases with increasing applied DC field, revealing that the wall vibrations become less dissipative when the number of domain walls decreases. This may again be due to the diminishing interaction between the domains likewise the case for the domain wall pinning/unpinning contribution. The value of the dissipation factor at zero bias field is 0.29 showing that, compared to the overall permittivity, the domain wall vibrations are also a very dissipative phenomenon.

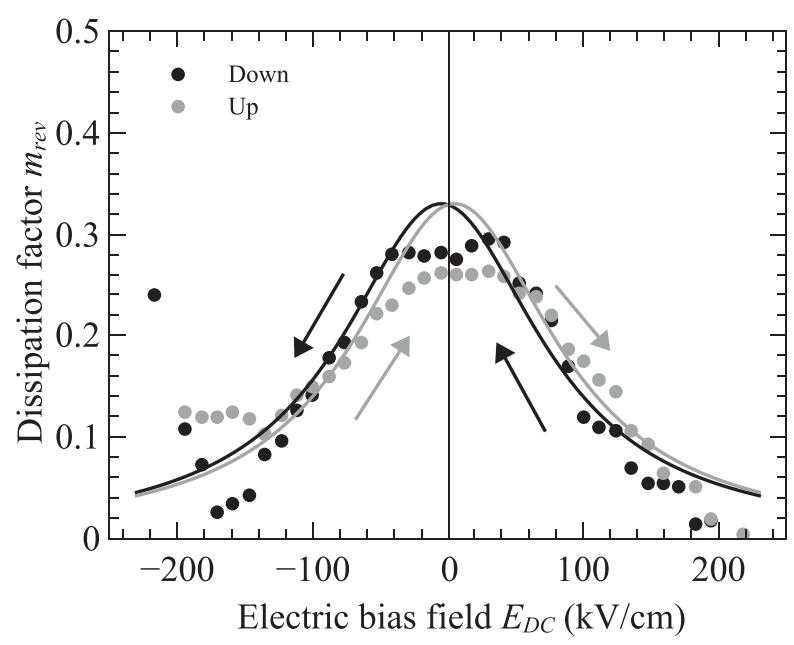

FIG. 10. Dissipation factor of the domain wall vibration contribution to the permittivity as a function of the applied DC field at $100 \mathrm{kHz}$.

Again, the Lorentzian fit has been used, and the coefficients are given in Table I. The main difference to domain wall pinning/unpinning is the infinite field value $A_{\infty-\text { mrev }}$ which seems to be zero, or at least very small. This signifies that the dissipative behavior of the domain vibrations is mainly due to the interaction between the domain walls. When there is no influence, the phenomenon is not dissipative, or the dissipation is negligible.

\section{Lattice contribution}

The lattice contribution to the material's permittivity is presented in Fig. 11. The behavior is very similar to what is observed for the overall permittivity previously presented and clearly is the dominant contribution. As already seen before, the real and imaginary parts decrease when the applied DC field increases. The same Lorentzian fits have been used. The coefficients are very close to those obtained from the fitting of the overall permittivity, especially for the real part (Table I). This confirms the fact that the lattice contribution is predominant in the case of the studied material. The weight of the different contributions will be discussed in Section III C. The value of the real part of the permittivity for an infinite DC field $A_{\infty-l}^{\prime}$ is not zero which signifies that, if the Lorentzian fit is the appropriate function for describing the evolution, the tunability has a limit value below $100 \%$ even if the applied field tends to infinity. The limit value $A_{\infty-l}^{\prime}$ may depend on the material, the stoichiometry, the film thickness, etc. This effect can be seen in Refs. 11 and 19 where different films have been measured at high DC field. In those papers, even if the unbiased permittivity varies, the value at high bias fields seems to be constant, which signifies that the limit value of the permittivity seems to be attained. In our case, the limit value is not null for the imaginary part, too.

The limit values for the real and the imaginary parts of the lattice contribution are identical to these for the total permittivity, which well reflects the fact that the domain wall contributions, vibration and pinning/unpinning, tend to zero when the applied bias field is infinite. These infinite values 


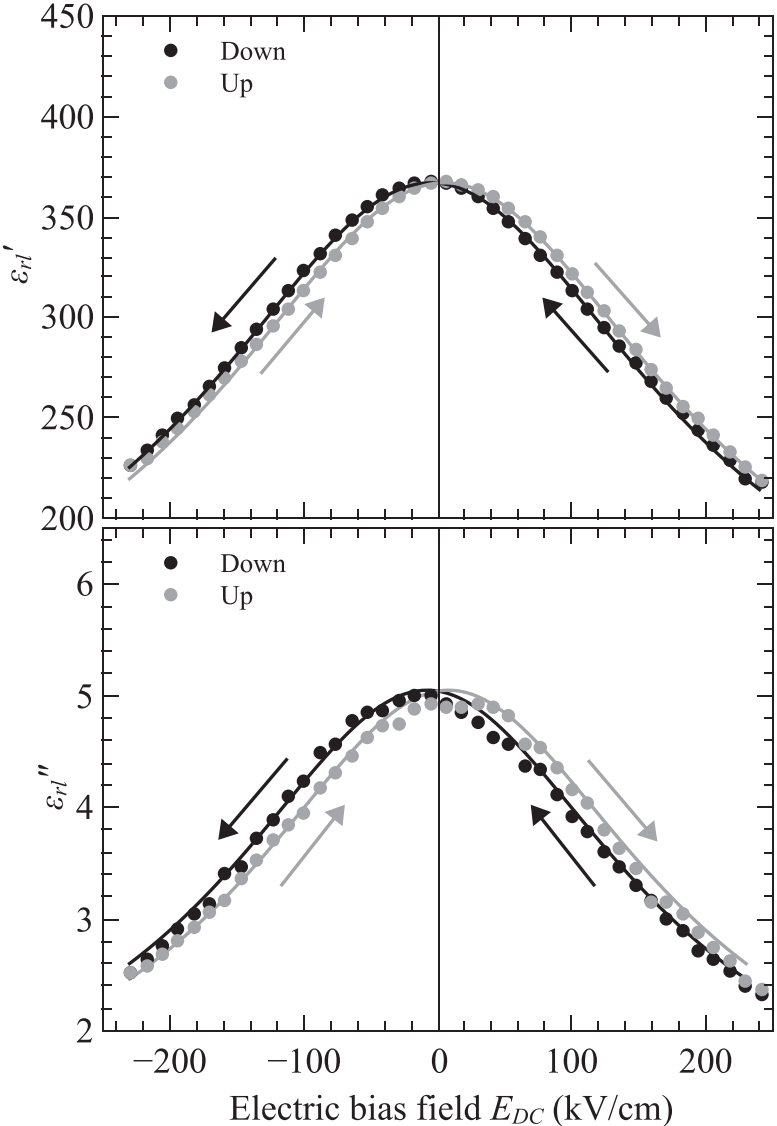

FIG. 11. Real and imaginary parts of the lattice contribution to the permittivity as a function of the applied DC field at $100 \mathrm{kHz}$.

$A_{\infty-l}$ are close to those of non tunable high-k materials and represent the intrinsic contribution to the permittivity of the material, without a configuration into domains.

The dissipation factor of the lattice contribution as a function of the applied bias field is given in Fig. 12. The evolution is very similar to the other dissipation factors; thus, the same fit has been used, and the coefficients are reported in Table I. As in the case of domain wall pinning, the dissipation tends to a non-zero value when the DC bias field goes

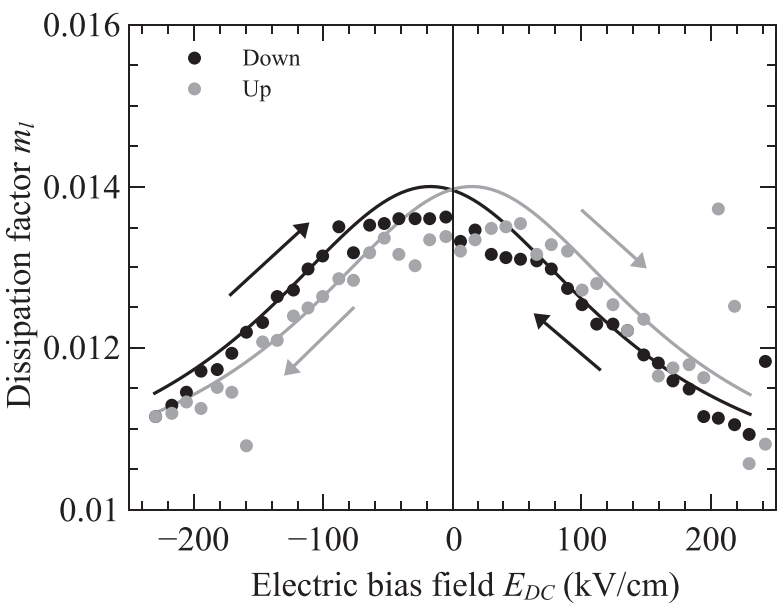

FIG. 12. Dissipation factor of the lattice contribution to the permittivity as a function of the applied DC field at $100 \mathrm{kHz}$. to infinity, signifying that the contribution of the lattice will be always lossy even at very high DC fields.

The tunability of the lattice contribution is $40.7 \%$, and the maximum losses are $m_{l}=0.014$. Those values are slightly smaller than these observed for the overall properties, also indicating that other phenomena participate to permittivity and losses, namely, the domain wall motion contributions presented before. The figure of merit of the lattice contribution $\mathrm{FoM}_{l}$ is 29.4 which is 10 times larger than that of the domain wall motion.

\section{On the importance of each contribution}

To better compare the contribution of each phenomenon to the overall properties of the ferroelectric thin film, the respective parts of losses and tunability are summarized in Table II for an AC field of $12 \mathrm{kV} / \mathrm{cm}$. To compute the weight of each contribution, we first calculate the weight of the lattice, which permits us to obtain the total weight of the domain motions, the complement to 100 . Then, this value is separated into the weight of the two contributions of the domain wall motion, vibration and pinning/unpinning. This method is used for the permittivity and losses where the direct sum cannot be used due to the presence of the square root. For the calculation of the percentage of a contribution in the overall tunability, an equivalent tunability $n_{r-\varepsilon}^{e q}$ is used. It represents the tunability of a contribution by taking into account its weight into the overall permittivity. It is computed using the classical formula (2) but by supposing only the considered contribution is tunable and the other contributions are kept constant.

For the studied material, the domain wall motion contribution to the real part of the permittivity is very weak, and the lattice contribution represents $98 \%$ of the total permittivity. As the domain walls are very sensitive to the DC field, the domain wall pinning/unpinning contributes slightly more to the tunability $(2.6 \%)$ than to the permittivity $(1.3 \%)$. The pinning/unpinning contribution to the total losses, however, represents $12.5 \%$. This is due to the fact that the domain wall motion is a very dissipative phenomenon. To obtain a high FoM, it is hence necessary to limit the contributions of the domain wall motion, even if the tunability is rather high. Moreover, limiting these contributions presents an advantage for the frequency stability of the material, as domain wall pinning/unpinning is very frequency dependent contrary to the lattice contribution. ${ }^{7,16,17}$

To reflect the weight of the domain wall contributions in the overall material's properties, the tunability and the FoM are reported in Fig. 13 as a function of the AC field. The tunability slightly increases from $40.8 \%$ at zero AC field to

TABLE II. Weight of the different contributions to the material's overall permittivity, losses, and tunability for an AC field of $12 \mathrm{kV} / \mathrm{cm}$.

\begin{tabular}{lccc}
\hline \hline Contribution & Permittivity (\%) & Losses (\%) & Tunability (\%) \\
\hline Lattice & 98.3 & 82.8 & 97.2 \\
Vibration & 0.4 & 4.7 & 0.2 \\
Pinning/unpinning & 1.3 & 12.5 & 2.6 \\
\hline \hline
\end{tabular}




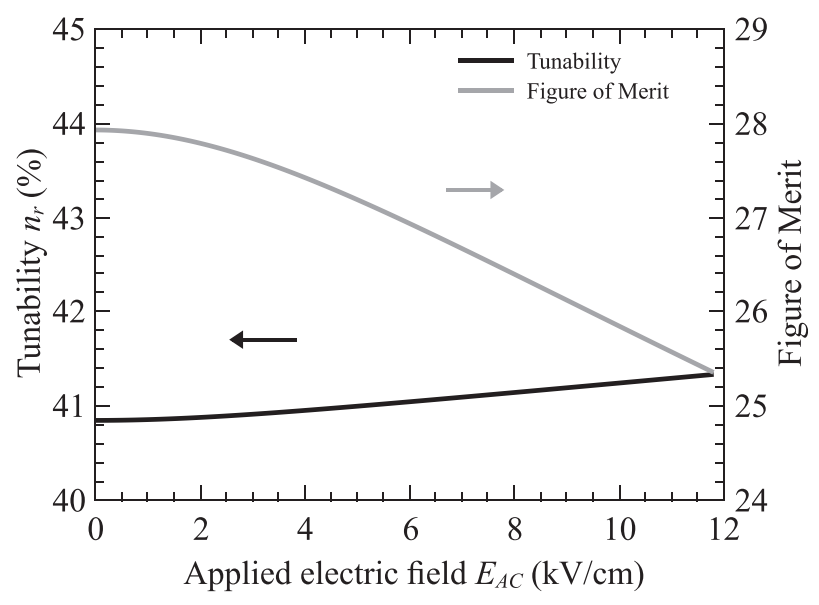

FIG. 13. Tunability and FoM at $100 \mathrm{kHz}$ under $230 \mathrm{kV} / \mathrm{cm}$ applied DC field as a function of the applied AC bias field.

$41.3 \%$ at $12 \mathrm{kV} / \mathrm{cm}$ due to the domain wall pinning/unpinning contribution which is AC field dependent. As this phenomenon is very dissipative, the losses also increase, which result in a decrease of the FoM. This behavior is similar to what has been reported elsewhere ${ }^{5}$ and clearly reveals that the domain wall motion should be limited in order to obtain a high FoM. The FoM diminishes from 27.9 at zero AC field to 25.4 at $12 \mathrm{kV} / \mathrm{cm}$. The decrease of the FoM also shows that the AC field use for the characterization is important and has to be identical to that used for application.

\section{CONCLUSION}

The complex permittivity of a $\mathrm{Ba}_{0.80} \mathrm{Sr}_{0.20} \mathrm{TiO}_{3}$ thin film doped with $1 \%$ manganese has been studied as a function of the AC measuring field for different DC bias fields. This allows to determine the weight of the different contributions to the material's permittivity, losses, and tunability.

Due to the coalescence of the domains, the two contributions of the domain wall motions (vibrations and pinning/ unpinning) decrease when the applied DC field increases. This may be also influenced by the interaction between the domain walls itself which increases the dissipation factor when the number of domain walls is important.

The observed tunabilities of the domain wall vibration and pinning/unpinning phenomena are $77 \%$ and $86 \%$, respectively, which are very high with respect to the lattice tunability (41\%). This reflects the strong influence of the DC bias field on the domain wall density. However, as the dissipation factor is, respectively, 0.29 and 0.2 for vibration and pinning/unpinning, a high domain wall contribution to the overall material's permittivity it is not an advantage. The figure of merit associated to the domain wall contributions is
2.7 for vibration and 4.14 for pinning/unpinning, which is very poor compared to the bulk FoM value (29.4). This confirms that the domain wall contributions are very dissipative phenomena and shows, even if there tunabilities are high, these contributions should be reduced in order to obtain a high overall FoM for the material. A high FoM is necessary when envisaging utilization of the ferroelectric thin film material for microwave device applications.

Finally, we also show that the amplitude of the AC characterizing field is important when determining the material's complex permittivity, since the dielectric properties and hence the FoM are influenced by this field.

\section{ACKNOWLEDGMENTS}

The authors would like to acknowledge the financial support of the CNRS (Centre National de la Recherche Scientifique) and the French Region "Pays de la Loire."

${ }^{1}$ K. Nadaud, R. Gillard, E. Fourn, C. Borderon, and H. Gundel, in 9th European Conference on Antennas and Propagation (EuCAP) (2015).

${ }^{2}$ J. Hai, M. Patterson, D. Brown, Z. Chenhao, P. KuanChang, G. Subramanyam, D. Kuhl, K. Leedy, and C. Cerny, IEEE Trans. Antennas Propag. 60, 3111 (2012).

${ }^{3}$ A. Ghalem, F. Ponchel, D. Remiens, and T. Lasri, in Proceedings of the IEEE International Symposium on Applications of Ferroelectrics (2013), pp. 252-256.

${ }^{4}$ C. Borderon, R. Renoud, M. Ragheb, and H. W. Gundel, Appl. Phys. Lett. 98, 112903 (2011)

${ }^{5}$ R. Renoud, C. Borderon, and H. Gundel, IEEE Trans. Ultrason., Ferroelectr., Freq. Control 58, 1975 (2011).

${ }^{6}$ O. Boser, J. Appl. Phys. 62, 1344 (1987).

${ }^{7}$ K. Nadaud, C. Borderon, R. Renoud, and H. W. Gundel, J. Appl. Phys. 117, 084104 (2015)

${ }^{8}$ D. Dimos, M. V. Raymond, R. W. Schwartz, H. N. Al-Shareef, and C. H. Mueller, J. Electroceram. 12, 145 (1997).

${ }^{9}$ K. Nadaud, C. Borderon, R. Gillard, E. Fourn, R. Renoud, and H. W. Gundel, Thin Solid Films 591, 90 (2015).

${ }^{10}$ M.-C. Chiu, Y.-C. Lee, and F.-S. Shieu, J. Electrochem. Soc. 152, F194 (2005).

${ }^{11}$ D. Levasseur, E. Bouyssou, R. De Paolis, A. Rousseau, F. Coccetti, G. Guegan, S. Payan, and M. Maglione, J. Phys.: Condens. Matter 25, 495901 (2013).

${ }^{12}$ J.-G. Cheng, X.-J. Meng, B. Li, J. Tang, S.-L. Guo, J.-H. Chu, M. Wang, H. Wang, and Z. Wang, Appl. Phys. Lett. 75, 2132 (1999).

${ }^{13}$ L. Wang, J. Yu, Y. Wang, G. Peng, F. Liu, and J. Gao, J. Appl. Phys. 101, 104505 (2007).

${ }^{14}$ J. Y. Jo, H. S. Han, J.-G. Yoon, T. K. Song, S.-H. Kim, and T. W. Noh, Phys. Rev. Lett. 99, 267602 (2007).

${ }^{15}$ D. Damjanovic, Rep. Prog. Phys. 61, 1267 (1998).

${ }^{16}$ D. V. Taylor and D. Damjanovic, J. Appl. Phys. 82, 1973 (1997).

${ }^{17}$ W. Zhu, I. Fujii, W. Ren, and S. Trolier-McKinstry, J. Appl. Phys. 109, 064105 (2011).

${ }^{18}$ J. E. García, R. Pérez, and A. Albareda, J. Phys.: Condens. Matter 17, 7143 (2005).

${ }^{19}$ N. K. Pervez, P. J. Hansen, and R. A. York, Appl. Phys. Lett. 85, 4451 (2004). 